\title{
Brief cognitive-behavioural therapy for patients in the community with schizophrenia: Randomised controlled trial in Beijing, China - Expression of concern
}

Zhi-Hua Guo, Zhan-Jiang Li, Yun Ma, Jing Sun, Jun-Hua Guo, Wen-Xiu Li, Zhi-Qiang Wang, Hui-Li Xu, Roger M. K. Ng, Douglas Turkington and David Kingdon

DOI: https://doi.org/10.1192/bjp.bp.116.183285, Published by Cambridge University Press, vol. 210, Issue 3, March 2017, pp. 223-229

Concerns have been raised regarding inconsistencies between the British Journal of Psychiatry article 'Brief cognitive-behavioural therapy for patients in the community with schizophrenia: Randomised controlled trial in Beijing, China' and the associated retrospective clinical trial registration (ChiCTR-TRC-13003709).

The authors have responded to these concerns, acknowledging that some errors were made. The case has also been reviewed by the COPE forum. However, a final resolution has not yet been reached, and the matter is being evaluated by the Senior Editorial Committee.

This Expression of Concern should not be considered as a statement regarding validity of the work but rather as a notification to readers, and an intent to provide additional information as it becomes available.

Zhi-Hua Guo, Zhan-Jiang Li, Yun Ma, Jing Sun, Jun-Hua Guo, Wen-Xiu Li,
Zhi-Qiang Wang, Hui-Li Xu, Roger M. K. Ng, Douglas Turkington, David Kingdon

\section{Reference}

Zhi-Hua Guo, Zhan-Jiang Li, Yun Ma, Jing Sun, Jun-Hua Guo, Wen-Xiu Li, Zhi-Qiang Wang, Hui-Li Xu, Roger M. K. Ng, Douglas Turkington, David Kingdon, 'Brief cognitivebehavioural therapy for patients in the community with schizophrenia: Randomised controlled trial in Beijing, China', British Journal of Psychiatry, Volume 210, Issue 3 March 2017, pp. 223-229 